\author{
Youn-Sha Chan \\ Department of Computer and Mathematical \\ Sciences, \\ University of Houston-Downtown, \\ One Main Street, \\ Houston, TX 77002 \\ Glaucio H. Paulino \\ Department of Civil and Environmental \\ Engineering, \\ University of Illinois, \\ 2209 Newmark Laboratory, \\ 205 North Mathews Avenue, \\ Urbana, IL 61801 \\ Albert C. Fannjiang \\ Department of Mathematics, \\ University of California, \\ Davis, CA 95616
}

\section{Gradient Elasticity Theory for Mode III Fracture in Functionally Graded Materials-Part II: Crack Parallel to the Material Gradation}

A Mode-III crack problem in a functionally graded material modeled by anisotropic strain-gradient elasticity theory is solved by the integral equation method. The gradient elasticity theory has two material characteristic lengths $\ell$ and $\ell^{\prime}$, which are responsible for volumetric and surface strain-gradient terms, respectively. The governing differential equation of the problem is derived assuming that the shear modulus $G$ is a function of $x$, i.e., $G=G(x)=G_{0} e^{\beta x}$, where $G_{0}$ and $\beta$ are material constants. A hypersingular integrodifferential equation is derived and discretized by means of the collocation method and a Chebyshev polynomial expansion. Numerical results are given in terms of the crack opening displacements, strains, and stresses with various combinations of the parameters $\ell, \ell^{\prime}$, and $\beta$. Formulas for the stress intensity factors, $K_{I I I}$, are derived and numerical results are provided. [DOI: 10.1115/1.2912933]

\section{Introduction}

This work is a continuation of the paper on "Gradient Elasticity Theory for Mode III Fracture in Functionally Graded MaterialsPart I: Crack Perpendicular to the Material Gradation" by Paulino et al. [1] (hereinafter referred to as Part I). In Part I, the authors considered a plane elasticity problem in which the medium contains a finite crack on the $y=0$ plane and the material gradation is perpendicular to the crack. In "Part II," the material gradation is parallel to the crack (see Fig. 1). In Part I, the shear modulus $G$ (that rules the material gradation) is a function of $y$ only, $G$ $\equiv G(y)=G_{0} e^{\gamma y}$; while in Part II, it is a function of $x$, i.e., $G$ $\equiv G(x)=G_{0} e^{\beta x}$. An immediate consequence of the difference in geometry, which is indicated in Fig. 1, is that the location of the crack in Part I is rather irrelevant to the problem and thus can be shifted so that the center is at the origin point $(0,0)$. On the other hand, if the material gradation is parallel to the crack, then the location of the crack is pertinent to the solution of the problem.

The method of solution is essentially the same in both Parts I and II, i.e., the integral equation method. However, because of differences in the geometrical configurations, some changes are expected. For instance, in Part I, the crack opening displacement profile is symmetric with respect to the $y$-axis, while in Part II, the symmetry of the crack profiles no longer exists. Thus, some interesting questions arise.

- How are the crack opening displacement profiles affected by the gradient elasticity and the gradation of the material?

- How are the stresses influenced under the gradient elasticity?

- How are the stress intensity factors (SIFs) calculated?

- How do the results compare to the classical linear elastic fracture mechanics (LEFM)?

We will address all the above questions. The remainder of the paper is organized as follows. First, the constitutive equations of anisotropic gradient elasticity for nonhomogeneous materials subjected to antiplane shear deformation are given. Then, the govern-

Contributed by the Applied Mechanics Division of ASME for publication in the Journal of ApPlied Mechanics. Manuscript received February 23, 2007; final manuscript received February 27, 2007; published online August 21, 2008. Review conducted by Robert M. McMeeking. ing partial differential equations (PDEs) are derived, and the Fourier transform method is introduced and applied to convert the governing PDE into an ordinary differential equation (ODE). Afterward, the crack boundary value problem is described, and a specific complete set of boundary conditions is given. The governing hypersingular integrodifferential equation is derived and discretized using the collocation method. Next, various relevant aspects of the numerical discretization are described in detail. Subsequently, numerical results are given, conclusions are inferred, and potential extensions of this work are discussed. One appendix, providing the hierarchy of the PDEs and the corresponding integral equations, supplements the paper.

\section{Constitutive Equations of Gradient Elasticity}

A schematic demonstration of continuously graded microstructure in functionally graded materials (FGMs) is illustrated by Fig. 2. The linkage between gradient elasticity and graded materials within the framework of fracture mechanics and its related work has been addressed in Part I. For the sake of completeness, the notation and constitutive equations of gradient elasticity for an antiplane shear crack in a FGM are briefly given in this section and particularized to the case of an exponentially graded material along the $x$-direction.

For an antiplane shear problem, the relevant displacement components are as follows:

$$
u=v=0, \quad w=w(x, y)
$$

and the nontrivial strains are as follows:

$$
\epsilon_{x z}=\frac{1}{2} \frac{\partial w}{\partial x}, \quad \epsilon_{y z}=\frac{1}{2} \frac{\partial w}{\partial y}
$$

The constitutive equations of gradient elasticity for FGMs are $[1,2]$ as follows:

$$
\begin{aligned}
\boldsymbol{\sigma}_{i j}= & \lambda(x) \epsilon_{k k} \delta_{i j}+2 G(x)\left(\epsilon_{i j}-\ell^{2} \nabla^{2} \epsilon_{i j}\right)-\ell^{2}\left[\partial_{k} \lambda(x)\right]\left(\partial_{k} \epsilon_{l l}\right) \delta_{i j} \\
& -2 \ell^{2}\left[\partial_{k} G(x)\right]\left(\partial_{k} \epsilon_{i j}\right) \\
\tau_{i j}= & \lambda(x) \epsilon_{k k} \delta_{i j}+2 G(x) \epsilon_{i j}+2 \ell^{\prime} \nu_{k}\left[\epsilon_{i j} \partial_{k} G(x)+G(x) \partial_{k} \epsilon_{i j}\right]
\end{aligned}
$$




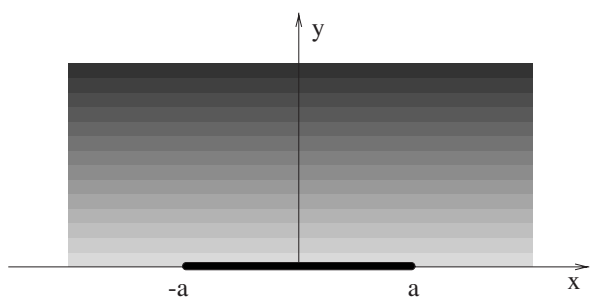

Material gradation perpendicular to the crack.

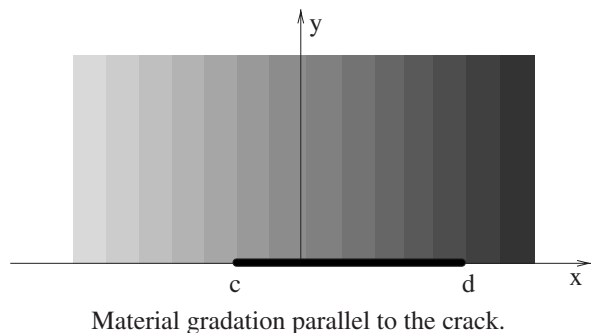

Fig. 1 A geometric comparison of the material gradation with respect to the crack location

$$
\boldsymbol{\mu}_{k i j}=2 \ell^{\prime} \nu_{k} G(x) \epsilon_{i j}+2 \ell^{2} G(x) \partial_{k} \epsilon_{i j}
$$

where $\ell$ is the characteristic length of the material responsible for volumetric strain-gradient terms, $\ell^{\prime}$ is responsible for surface strain-gradient terms, $\boldsymbol{\sigma}_{i j}$ is the stress tensor, and $\boldsymbol{\mu}_{i j k}$ is the couple-stress tensor. The Lamé moduli $\lambda \equiv \lambda(x)$ and $G \equiv G(x)$ are assumed to be functions of $x$. Moreover, $\partial_{k}=\partial / \partial x_{k}$. The parameter $\ell^{\prime}$ is associated with surfaces and $\nu_{k}, \partial_{k} \nu_{k}=0$, is a director field equal to the unit outer normal $n_{k}$ on the boundaries.

For a Mode-III problem, the constitutive equations above become

$$
\begin{gathered}
\sigma_{x x}=\sigma_{y y}=\sigma_{z z}=0, \quad \sigma_{x y}=0 \\
\sigma_{x z}=2 G(x)\left(\epsilon_{x z}-\ell^{2} \nabla^{2} \epsilon_{x z}\right)-2 \ell^{2}\left[\partial_{x} G(x)\right]\left(\partial_{x} \epsilon_{x z}\right) \neq 0 \\
\sigma_{y z}=2 G(x)\left(\epsilon_{y z}-\ell^{2} \nabla^{2} \epsilon_{y z}\right)-2 \ell^{2}\left[\partial_{x} G(x)\right]\left(\partial_{x} \epsilon_{y z}\right) \neq 0 \\
\mu_{x x z}=2 G(x) \ell^{2} \partial \epsilon_{x z} / \partial x
\end{gathered}
$$

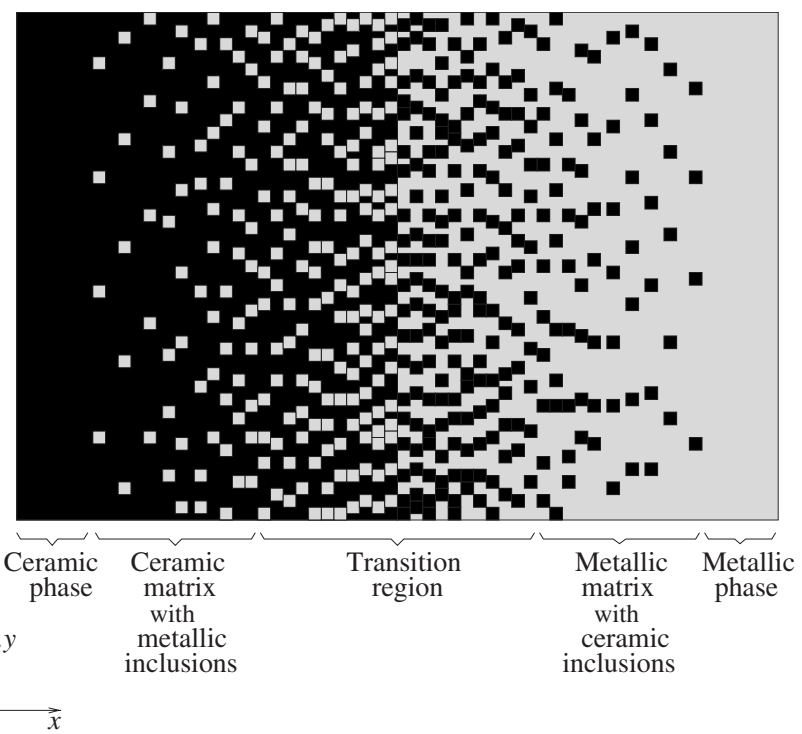

Fig. 2 A schematic illustration of a continuously graded microstructure in FGMs

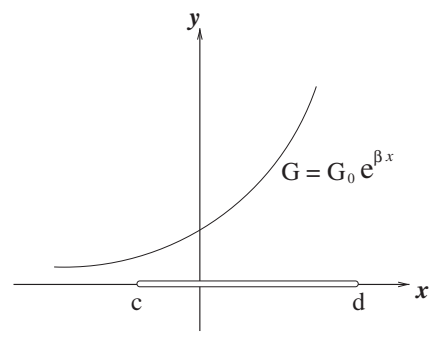

Fig. 3 Geometry of the crack problem and material gradation

$$
\begin{gathered}
\mu_{x y z}=2 G(x) \ell^{2} \partial \epsilon_{y z} / \partial x \\
\mu_{y x z}=2 G(x)\left(\ell^{2} \partial \epsilon_{x z} / \partial y-\ell^{\prime} \epsilon_{x z}\right) \\
\mu_{y y z}=2 G(x)\left(\ell^{2} \partial \epsilon_{y z} / \partial y-\ell^{\prime} \epsilon_{y z}\right)
\end{gathered}
$$

If $G$ is constant, i.e., the material is homogeneous, then the constitutive equations $[3,4]$ are $^{1}$ as follows:

$$
\begin{gathered}
\sigma_{x x}=\sigma_{y y}=\sigma_{z z}=0, \quad \sigma_{x y}=0 \\
\sigma_{x z}=2 G\left(\epsilon_{x z}-\ell^{2} \nabla^{2} \epsilon_{x z}\right) \neq 0 \\
\sigma_{y z}=2 G\left(\epsilon_{y z}-\ell^{2} \nabla^{2} \epsilon_{y z}\right) \neq 0 \\
\mu_{x x z}=2 G \ell^{2} \partial \epsilon_{x z} / \partial x \\
\mu_{x y z}=2 G \ell^{2} \partial \epsilon_{y z} / \partial x \\
\mu_{y x z}=2 G\left(\ell^{2} \partial \epsilon_{x z} / \partial y-\ell^{\prime} \epsilon_{x z}\right) \\
\mu_{y y z}=2 G\left(\ell^{2} \partial \epsilon_{y z} / \partial y-\ell^{\prime} \epsilon_{y z}\right)
\end{gathered}
$$

It is worth to point out that each of the total stresses $\sigma_{x z}$ and $\sigma_{y z}$ in Eq. (6) has an extra term than the ones in (7) due to the material gradation interplay with the strain-gradient effect [2].

\section{Governing Partial Differential Equation and Bound- ary Conditions}

By imposing the only nontrivial equilibrium equation

$$
\frac{\partial \sigma_{x z}}{\partial x}+\frac{\partial \sigma_{y z}}{\partial y}=0
$$

the following PDE is obtained:

$$
\begin{array}{r}
\frac{\partial}{\partial x}\left[G(x)\left(\frac{\partial w}{\partial x}-\ell^{2} \nabla^{2} \frac{\partial w}{\partial x}\right)\right]+\frac{\partial}{\partial y}\left[G(x)\left(\frac{\partial w}{\partial y}-\ell^{2} \nabla^{2} \frac{\partial w}{\partial y}\right)\right] \\
-\ell^{2}\left[\frac{\partial^{2} G(x)}{\partial x^{2}} \frac{\partial^{2} w}{\partial x^{2}}+\frac{\partial G(x)}{\partial x} \frac{\partial^{3} w}{\partial x^{3}}+\frac{\partial G(x)}{\partial x} \frac{\partial^{3} w}{\partial x \partial y^{2}}\right]=0
\end{array}
$$

If the shear modulus $G$ is assumed as an exponential function of $x$ (see Fig. 3),

$$
G=G(x)=G_{0} e^{\beta x}
$$

then PDE (9) can be simplified as

$$
-\ell^{2} \nabla^{4} w-2 \beta \ell^{2} \nabla^{2} \frac{\partial w}{\partial x}+\nabla^{2} w-\beta^{2} \ell^{2} \frac{\partial^{2} w}{\partial x^{2}}+\beta \frac{\partial w}{\partial x}=0
$$

or

${ }^{1}$ According to the geometry of the problem (see Fig. 3), it is the upper half-plane that is considered in the formulation. The crack is sitting on the $x$-axis, which is on the boundary of the upper half-plane. Thus, the outward unit normal should be $(0,-1,0)$, and not $(0,1,0)$. Based on Eq. (5), or the last equation in Eq. (5) of Ref. [4], the sign in front of $\ell^{\prime}$ in the expression for both $\mu_{y x z}$ and $\mu_{y y z}$ should be "-" instead of "+." 
Table 1 Governing PDEs in antiplane shear problems

\begin{tabular}{ccl}
\hline \hline Cases & Governing PDE & References \\
\hline$\ell=0, \beta=0$ & Laplace equation: & Standard textbooks \\
$\ell=0, \beta \neq 0$ & $\nabla^{2} w=0$ & \\
$\ell \neq 0, \beta=0$ & $\begin{array}{c}\text { Perturbed Laplace equation: } \\
\left(\nabla^{2}+\beta \partial / \partial x\right) w=0\end{array}$ & Erdogan [7] \\
& $\begin{array}{c}\text { Helmholtz-Laplace equation: } \\
\left(1-\ell^{2} \nabla^{2}\right) \nabla^{2} w=0\end{array}$ & $\begin{array}{l}\text { Vardoulakis et al. [4] } \\
\text { Fannjiang et al. [5] } \\
\ell \neq 0, \beta \neq 0\end{array}$ \\
& Equation (11): & $\begin{array}{l}\text { Zhang et al. [8] } \\
\text { Studied in this paper }\end{array}$ \\
& $\left(1-\beta \ell^{2} \frac{\partial}{\partial x}-\ell^{2} \nabla^{2}\right)\left(\nabla^{2}+\beta \frac{\partial}{\partial x}\right) w=0$ &
\end{tabular}

$$
\left(1-\beta \ell^{2} \frac{\partial}{\partial x}-\ell^{2} \nabla^{2}\right)\left(\nabla^{2}+\beta \frac{\partial}{\partial x}\right) w=0
$$

which is the governing PDE solved in the present paper.

It may be seen, from a viewpoint of perturbation, that PDE (12) can be expressed in an operator form, i.e.,

$$
\mathrm{H}_{\beta} \mathrm{L}_{\beta} w=0, \quad \mathrm{H}_{\beta}=1-\beta \ell^{2} \frac{\partial}{\partial x}-\ell^{2} \nabla^{2}, \quad \mathrm{~L}_{\beta}=\nabla^{2}+\beta \frac{\partial}{\partial x}
$$

where $\mathrm{H}_{\beta}$ is the perturbed Helmholtz operator, $\mathrm{L}_{\beta}$ is the perturbed Laplacian operator, and the two operators commute $\left(\mathrm{H}_{\beta} \mathrm{L}_{\beta}\right.$ $=\mathrm{L}_{\beta} \mathrm{H}_{\beta}$. By sending $\beta \rightarrow 0$, we get the PDE [4,5]

$$
\left(1-\ell^{2} \nabla^{2}\right) \nabla^{2} w=0 \quad \text { or } \mathrm{HL} w=0
$$

where the Helmholtz operator $\mathrm{H}=1-\ell^{2} \nabla^{2}$ and the Laplacian operator $L=\nabla^{2}$ are invariant under any change of variables by rotations and translations. FGM creates the perturbation and ruins the invariance. However, the perturbing term " $-\beta \ell^{2}(\partial / \partial x)$ " in $\mathrm{L}_{\beta}$, which is not purely caused by the gradation of the material, involves both the gradation parameter $\beta$ and the characteristic length $\ell$ (the product of $\beta$ and $\ell^{2}$ ). It can be interpreted as a consequence of the interaction of the material gradation and the strain-gradient effect [2].

If we let $\ell \rightarrow 0$ alone, then the perturbed Helmholtz differential operator $\mathrm{H}_{\beta}$ will become the identity operator, and one reduces PDE (12) to

$$
\left(\nabla^{2}+\beta \frac{\partial}{\partial x}\right) w=0
$$

the perturbed Laplace equation, which is the PDE that governs the Mode-III crack problem for nonhomogeneous materials with shear modulus $G(x)=G_{0} e^{\beta x}[6,7]$. The limit of sending $\ell \rightarrow 0$ will lower the fourth order PDE (11) to a second order one (Eq. (15)), and a singular perturbation is expected. By taking both limits $\beta$ $\rightarrow 0$ and $\ell \rightarrow 0$, one obtains the harmonic equation for classical elasticity. Various combinations of parameters $\ell$ and $\beta$ with the corresponding governing PDE are listed in Table 1.

One may notice that in the governing PDE (12), there is no surface term parameter $\ell^{\prime}$ involved. However, $\ell^{\prime}$ does influence the solution through the boundary conditions. By the principle of virtual work, the following boundary conditions can be derived and are adopted in this paper:

$$
\begin{gathered}
\sigma_{y z}(x, 0)=p(x), \quad x \in(c, d) \\
w(x, 0)=0, \quad x \notin(c, d) \\
\mu_{y y z}(x, 0)=0, \quad-\infty<x<+\infty
\end{gathered}
$$

The first two boundary conditions in Eq. (16) are from classical LEFM, and the last one, involving the couple-stress $\mu_{y y z}$, is needed for the higher order theory. This set of boundary condi- tions is the same as those adopted by Vardoulakis et al. [4] An alternative treatment of boundary conditions can be found in Ref. [9].

\section{Fourier Transform}

Let the Fourier transform be defined by

$$
\mathcal{F}(w)(\xi)=W(\xi)=\frac{1}{\sqrt{2 \pi}} \int_{-\infty}^{\infty} w(x) e^{i x \xi} d x
$$

Then, by the Fourier integral formula [10],

$$
\mathcal{F}^{-1}(W)(x)=w(x)=\frac{1}{\sqrt{2 \pi}} \int_{-\infty}^{\infty} W(\xi) e^{-i x \xi} d \xi
$$

where $\mathcal{F}^{-1}$ denotes the inverse Fourier transform. Now, let us assume that

$$
w(x, y)=\frac{1}{\sqrt{2 \pi}} \int_{-\infty}^{\infty} W(\xi, y) e^{-i x \xi} d \xi
$$

where $w(x, y)$ is the inverse Fourier transform of the function $W(\xi, y)$. By considering each term in Eq. (11) and using Eq. (19), one obtains

$$
\begin{gathered}
-\ell^{2} \nabla^{4} w=\frac{-\ell^{2}}{\sqrt{2 \pi}} \int_{-\infty}^{\infty}\left(\xi^{4} W(\xi, y)-2 \xi^{2} \frac{\partial^{2} W}{\partial y^{2}}+\frac{\partial^{4} W}{\partial y^{4}}\right) e^{-i x \xi} d \xi \\
-\beta \ell^{2} \nabla^{2} \frac{\partial w}{\partial x}=\frac{-\beta \ell^{2}}{\sqrt{2 \pi}} \int_{-\infty}^{\infty}\left(i \xi^{3} W(\xi, y)-i \xi \frac{\partial^{2} W}{\partial y^{2}}\right) e^{-i x \xi} d \xi \\
\nabla^{2} w=\frac{1}{\sqrt{2 \pi}} \int_{-\infty}^{\infty}\left(-\xi^{2} W(\xi, y)+\frac{\partial^{2} W}{\partial y^{2}}\right) e^{-i x \xi} d \xi \\
-\beta^{2} \ell^{2} \frac{\partial^{2} w(x, y)}{\partial x^{2}}=\frac{\beta^{2} \ell^{2}}{\sqrt{2 \pi}} \int_{-\infty}^{\infty} \xi^{2} W(\xi, y) e^{-i x \xi} d \xi \\
\beta \frac{\partial w(x, y)}{\partial x}=\frac{\beta}{\sqrt{2 \pi}} \int_{-\infty}^{\infty}(-i \xi) W(\xi, y) e^{-i x \xi} d \xi
\end{gathered}
$$

Equations (20)-(24) are added according to Eq. (11), and after simplification, the governing ODE is obtained:

$$
\begin{aligned}
& {\left[\ell^{2} \frac{d^{4}}{d y^{4}}-\left(2 \ell^{2} \xi^{2}+2 i \beta \ell^{2} \xi+1\right) \frac{d^{2}}{d y^{2}}\right.} \\
& \left.\quad+\left(\ell^{2} \xi^{4}+2 i \beta \ell^{2} \xi^{3}-\beta^{2} \ell^{2} \xi^{2}+\xi^{2}+i \beta \xi\right)\right] W=0
\end{aligned}
$$

\section{Solutions of the ODE}

The corresponding characteristic equation to the ODE (25) is

$$
\begin{aligned}
\ell^{2} \lambda^{4}- & \left(2 \ell^{2} \xi^{2}+2 i \beta \ell^{2} \xi+1\right) \lambda^{2} \\
+ & \left(\ell^{2} \xi^{4}+2 i \beta \ell^{2} \xi^{3}-\beta^{2} \ell^{2} \xi^{2}+\xi^{2}+i \beta \xi\right)=0
\end{aligned}
$$

which can be further factorized as

$$
\left[\ell^{2} \lambda^{2}-\left(1+i \beta \ell^{2} \xi+\ell^{2} \xi^{2}\right)\right]\left(\lambda^{2}-\xi^{2}-i \beta \xi\right)=0
$$

Clearly, the four roots $\lambda_{i}(i=1,2,3,4)$ of the polynomial (27) above can be written as

$$
\lambda_{1}=\frac{-1}{\sqrt{2}} \sqrt{\sqrt{\xi^{4}+\beta^{2} \xi^{2}}+\xi^{2}}-\frac{i}{\sqrt{2}} \frac{\beta \xi}{\sqrt{\sqrt{\xi^{4}+\beta^{2} \xi^{2}}+\xi^{2}}}
$$


Table 2 Roots $\lambda_{i}$ together with the corresponding mechanics theory and type of material

\begin{tabular}{|c|c|c|c|c|}
\hline Cases & $\begin{array}{l}\text { Number } \\
\text { of roots }\end{array}$ & Roots & $\begin{array}{l}\text { Mechanics theory } \\
\text { and type of material }\end{array}$ & References \\
\hline$\ell=0, \beta=0$ & 2 & $\pm|\xi|$ & $\begin{array}{l}\text { Classical LEFM, } \\
\text { homogeneous materials }\end{array}$ & Standard textbooks \\
\hline$\ell=0, \beta \neq 0$ & 2 & $\begin{array}{l}\lambda_{1} \text { and } \lambda_{2} \text { in Eqs. } \\
\text { (28) and (29), respectively }\end{array}$ & $\begin{array}{l}\text { Classical LEFM, } \\
\text { nonhomogeneous materials }\end{array}$ & Erdogan [7] \\
\hline$\ell \neq 0, \beta=0$ & 4 & $\pm|\xi|, \pm \sqrt{\xi^{2}+1 / \ell^{2}}$ & $\begin{array}{l}\text { Gradient theories, } \\
\text { homogeneous materials }\end{array}$ & $\begin{array}{l}\text { Vardoulakis et al. [4] } \\
\text { Fannjiang et al. [5] }\end{array}$ \\
\hline$\ell \neq 0, \beta \neq 0$ & 4 & $\begin{array}{l}\text { The four roots } \lambda_{1}-\lambda_{4} \\
\text { in Eqs. (28)-(31) }\end{array}$ & $\begin{array}{l}\text { Gradient theories, } \\
\text { nonhomogeneous materials }\end{array}$ & Studied in this paper \\
\hline
\end{tabular}

$$
\begin{aligned}
& \lambda_{2}= \frac{1}{\sqrt{2}} \sqrt{\sqrt{\xi^{4}+\beta^{2} \xi^{2}}+\xi^{2}}+\frac{i}{\sqrt{2}} \frac{\beta \xi}{\sqrt{\sqrt{\xi^{4}+\beta^{2} \xi^{2}}+\xi^{2}}} \\
& \lambda_{3}= \frac{-1}{\sqrt{2}} \sqrt{\sqrt{\left(\xi^{2}+1 / \ell^{2}\right)^{2}+\beta^{2} \xi^{2}}+\xi^{2}+1 / \ell^{2}} \\
&-\frac{i}{\sqrt{2}} \frac{\beta \xi}{\sqrt{\sqrt{\left(\xi^{2}+1 / \ell^{2}\right)^{2}+\beta^{2} \xi^{2}}+\xi^{2}+1 / \ell^{2}}} \\
& \lambda_{4}= \frac{1}{\sqrt{2}} \sqrt{\sqrt{\left(\xi^{2}+1 / \ell^{2}\right)^{2}+\beta^{2} \xi^{2}}+\xi^{2}+1 / \ell^{2}} \\
&+\frac{i}{\sqrt{2}} \frac{\beta \xi}{\sqrt{\sqrt{\left(\xi^{2}+1 / \ell^{2}\right)^{2}+\beta^{2} \xi^{2}}+\xi^{2}+1 / \ell^{2}}}
\end{aligned}
$$

If $\beta \rightarrow 0$, then the imaginary part of each root $\lambda_{i}(i=1, \ldots, 4)$ disappears. Thus, we have exactly the same roots found by Vardoulakis et al. [4] and Fannjiang et al. [5]. The root $\lambda_{1}$ corresponds to the solution of the perturbed harmonic equation $\nabla^{2} w$ $+\beta \partial w / \partial x=0$, and the root $\lambda_{3}$ agrees with the solution of the perturbed Helmholtz equation $\left(1-\beta \ell^{2} \partial / \partial x-\ell^{2} \nabla^{2}\right) w=0$. Various choices of parameters $\ell$ and $\beta$ with their corresponding mechanics theories and materials are listed in Table 2. In contrast to the four real roots found in Part I, the four roots here are all complex and admit a more complicated expression.

By the symmetry of the geometry, one can only consider the upper half-plane $(y>0)$. By taking account of the far-field boundary condition

$$
w(x, y) \rightarrow 0 \text { as } \sqrt{x^{2}+y^{2}} \rightarrow+\infty
$$

one can express the solution for $W(\xi, y)$ as

$$
W(\xi, y)=A(\xi) e^{\lambda_{1} y}+B(\xi) e^{\lambda_{3} y}
$$

where the nonpositive real part of $\lambda_{1}$ and $\lambda_{3}$ has been chosen to satisfy the far-field condition in the upper half-plane. Accordingly, the displacement $w(x, y)$ takes the form

$$
w(x, y)=\frac{1}{\sqrt{2 \pi}} \int_{-\infty}^{\infty}\left[A(\xi) e^{\lambda_{1} y}+B(\xi) e^{\lambda_{3} y}\right] e^{-i x \xi} d \xi
$$

Both $A(\xi)$ and $B(\xi)$ are determined by the boundary conditions.

\section{Hypersingular Integrodifferential Equation Ap- proach}

By substituting Eq. (34) into Eq. (6), we have

$$
\begin{aligned}
\sigma_{y z}(x, y) & =2 G(x)\left(\epsilon_{y z}-\ell^{2} \nabla^{2} \epsilon_{y z}\right)-2 \ell^{2}\left[\partial_{x} G(x)\right] \partial_{x} \epsilon_{y z} \\
& =\frac{G(x)}{\sqrt{2 \pi}} \int_{-\infty}^{\infty}\left[\lambda_{1} A(\xi) e^{\lambda_{1} y}\right] e^{-i x \xi} d \xi, \quad y \geq 0
\end{aligned}
$$

and

$$
\begin{aligned}
\mu_{y y z}(x, y)= & 2 G(x)\left(\ell^{2} \frac{\partial \epsilon_{y z}}{\partial y}-\ell^{\prime} \epsilon_{y z}\right) \\
= & \frac{G(x)}{\sqrt{2 \pi}} \int_{-\infty}^{\infty}\left\{\left(\ell^{2} \lambda_{1}^{2}-\ell^{\prime} \lambda_{1}\right) A(\xi) e^{\lambda_{1} y}\right. \\
& \left.+\left(\ell^{2} \lambda_{3}^{2}-\ell^{\prime} \lambda_{3}\right) B(\xi) e^{\lambda_{3} y}\right\} e^{-i x \xi} d \xi, \quad y \geq 0
\end{aligned}
$$

From the boundary condition in Eq. (16) imposed on the couplestress $\mu_{y y z}$ (i.e., $\mu_{y y z}(x, 0)=0$ for $-\infty<x<\infty$ ), one obtains the following relationship between $A(\xi)$ and $B(\xi)$ :

$$
B(\xi)=\frac{\ell^{\prime} \lambda_{1}-\ell^{2} \lambda_{1}^{2}}{\ell^{2} \lambda_{3}^{2}-\ell^{\prime} \lambda_{3}} A(\xi)=\rho(\beta, \xi) A(\xi)
$$

with

$$
\rho(\beta, \xi)=\frac{\ell^{\prime} \lambda_{1}-\ell^{2} \lambda_{1}^{2}}{\ell^{2} \lambda_{3}^{2}-\ell^{\prime} \lambda_{3}}=-\frac{\ell^{2} \xi^{2}+i \beta \ell^{2} \xi+\ell^{\prime} \sqrt{\xi^{2}+i \beta \xi}}{\ell^{\prime} \sqrt{\xi^{2}+i \beta \xi+1 / \ell^{2}}+\left(\ell^{2} \xi^{2}+i \beta \ell^{2} \xi+1\right)}
$$

Denote

$$
\begin{aligned}
\phi(x) & =\frac{\partial}{\partial x} w\left(x, 0^{+}\right)=\frac{1}{\sqrt{2 \pi}} \int_{-\infty}^{\infty}(-i \xi)[A(\xi)+B(\xi)] e^{-i x \xi} d \xi \\
& =\mathcal{F}^{-1}\{(-i \xi)[A(\xi)+B(\xi)]\}
\end{aligned}
$$

The second boundary condition in Eqs. (16) and (39) implies that

$$
\phi(x)=0, \quad x \notin[c, d]
$$

and

$$
\int_{c}^{d} \phi(x) d x=0
$$

which is the single-valuedness condition. By Eqs. (39) and (40), we obtain

$$
(-i \xi)[A(\xi)+B(\xi)]=\frac{1}{\sqrt{2 \pi}} \int_{-\infty}^{\infty} \phi(x) e^{i x \xi} d x=\frac{1}{\sqrt{2 \pi}} \int_{c}^{d} \phi(t) e^{i \xi t} d t
$$

By substituting Eq. (37) into Eq. (42) above, one gets

$$
A(\xi)=\frac{1}{\sqrt{2 \pi}}\left[\frac{1}{(-i \xi)[1+\rho(\beta, \xi)]}\right] \int_{c}^{d} \phi(t) e^{i \xi t} d t
$$

where

$$
\frac{1}{1+\rho(\beta, \xi)}=\frac{\left(\ell^{2} \xi^{2}+i \beta \ell^{2} \xi+1\right)+\ell^{\prime} \sqrt{\xi^{2}+i \beta \xi+1 / \ell^{2}}}{1+\ell^{\prime} \sqrt{\xi^{2}+i \beta \xi+1 / \ell^{2}}-\ell^{\prime} \sqrt{\xi^{2}+i \beta \xi}}
$$

By replacing the $A(\xi)$ in Eq. (35) by Eq. (43), one obtains the following integral equation in the limit $y \rightarrow 0^{+}$: 


$$
\begin{aligned}
\lim _{y \rightarrow 0^{+}} \sigma_{y z}(x, y)= & \lim _{y \rightarrow 0^{+}} \frac{G(x)}{2 \pi} \int_{-\infty}^{\infty} \lambda_{1}(\beta, \xi) \\
& \times\left[\frac{1}{(-i \xi)[1+\rho(\beta, \xi)]} \int_{c}^{d} \phi(t) e^{i \xi t} d t\right] e^{\lambda_{1} y} e^{-i x \xi} d \xi \\
= & \lim _{y \rightarrow 0^{+}} \frac{G(x)}{2 \pi} \int_{c}^{d} \phi(t) \int_{-\infty}^{\infty}\left[\frac{\lambda_{1}(\beta, \xi)}{(-i \xi)[1+\rho(\beta, \xi)]} e^{\lambda_{1} y}\right] \\
& \times e^{i(t-x) \xi} d \xi d t \\
= & \lim _{y \rightarrow 0^{+}} \frac{G}{2 \pi} \int_{c}^{d} \phi(t) \int_{-\infty}^{\infty} K(\xi, y) e^{i \xi(t-x)} d \xi d t \\
& -\infty<x<\infty
\end{aligned}
$$

with

$$
K(\xi, y)=\frac{\lambda_{1}(\beta, \xi)}{(-i \xi)[1+\rho(\beta, \xi)]} e^{\lambda_{1} y}
$$

Equation (46) is an expression for the stress $\sigma_{y z}(x, y)$ in the limit form of $y \rightarrow 0^{+}$, which is valid for $x \in(-\infty, \infty)$. Note that for the (first) boundary condition in Eq. (16), $\sigma_{y z}(x, 0)=p(x), x$ is restricted to the crack surface $(c, d)$. It is this boundary condition that leads to the governing hypersingular integrodifferential equation (see Eq. (55a) below). However, when SIFs are calculated, $x$ takes values outside of $(c, d)$, and the integral (46) is not singular (see Eq. (55b) below).

We split $K(\xi, y)$ into the singular part $K_{\infty}(\xi, y)$ and the nonsingular part $N(\xi, y)$ :

$$
K(\xi, y)=K_{\infty}(\xi, y)+N(\xi, y)
$$

where $K_{\infty}(\xi, y)$ is the nonvanishing part of the asymptotic expansion of $K(\xi, y)$ in the powers of $\xi$, as $|\xi| \rightarrow \infty$. When $y$ is set to be 0 , we have

$$
\begin{aligned}
K_{\infty}(\xi, 0)= & -i \ell^{2}|\xi| \xi-\frac{\ell^{\prime}}{2} i \xi+\frac{3 \beta \ell^{2}}{2}|\xi|+\frac{\ell^{\prime} \beta}{2} \\
& +\left[\left(\frac{\ell^{\prime}}{2 \ell}\right)^{2}+\frac{3 \ell^{2} \beta^{2}}{8}-1\right] \frac{i \xi}{|\xi|}
\end{aligned}
$$

Note that the real and the imaginary parts of $K_{\infty}(\xi, 0)$ given in Eq. (49) are even and odd functions of $\xi$, respectively.

In view of the following distributional convergence,

$$
\begin{gathered}
\int_{-\infty}^{\infty}\left[i \xi|\xi| e^{-|\xi| y}\right] e^{i(t-x) \xi} d \xi \stackrel{y \rightarrow 0^{+}}{\longrightarrow} \frac{4}{(t-x)^{3}} \\
\int_{-\infty}^{\infty}\left[|\xi| e^{-|\xi| y}\right] e^{i(t-x) \xi} d \xi \stackrel{y \rightarrow 0^{+}}{\longrightarrow} \frac{-2}{(t-x)^{2}} \\
\int_{-\infty}^{\infty}\left[i \xi e^{-|\xi| y}\right] e^{i(t-x) \xi} d \xi \stackrel{y \rightarrow 0^{+}}{\longrightarrow} 2 \pi \delta^{\prime}(t-x) \\
\int_{-\infty}^{\infty}\left[i \frac{|\xi|}{\xi} e^{-|\xi| y}\right] e^{i(t-x) \xi} d \xi \stackrel{y \rightarrow 0^{+}}{\longrightarrow} \frac{-2}{t-x} \\
\int_{-\infty}^{\infty}\left[1 e^{-|\xi| y}\right] e^{i(t-x) \xi} d \xi \stackrel{y \rightarrow 0^{+}}{\longrightarrow} 2 \pi \delta(t-x)
\end{gathered}
$$

with $\delta(x)$ being the Dirac delta function, we obtain the limit

$$
\begin{aligned}
\lim _{y \rightarrow 0^{+}} \int_{c}^{d} \int_{-\infty}^{\infty} K_{\infty}(\xi, y) e^{i \xi(t-x)} d \xi \phi(t) d t \\
=\frac{G}{\pi} \int_{c}^{d}\left\{\frac{-2 \ell^{2}}{(t-x)^{3}}-\frac{3 \beta \ell^{2}}{2(t-x)^{2}}+\frac{1-3 \beta^{2} \ell^{2} / 8-\left[\ell^{\prime} /(2 \ell)\right]^{2}}{t-x}\right. \\
\quad+k(x, t)\} \phi(t) d t+\frac{\ell^{\prime}}{2} \phi^{\prime}(x)+\frac{\beta \ell^{\prime}}{2} \phi(x)=p(x), \quad c<x<d \\
=\frac{1}{\pi} \int_{c}^{d}\left\{\frac{-2 \ell^{2}}{(t-x)^{3}}-\frac{3 \beta \ell^{2}}{2(t-x)^{2}}+\frac{1-3 \ell^{2} \beta^{2} / 8-\left[\ell^{\prime} /(2 \ell)\right]^{2}}{t-x}\right. \\
\quad+k(x, t)\} \phi(t) d t, \quad x<c \quad \text { or } x>d
\end{aligned}
$$

where $f$ denotes the finite-part integral [11], and the regular kernel $k(x, t)$ is given by

$$
k(x, t)=\int_{0}^{\infty} N(\xi, 0) e^{i(t-x) \xi} d \xi
$$

Equation (55a) is a Fredholm integral equation of the second kind with the cubic and Cauchy singular kernels.

\section{Numerical Solution}

To numerically solve the unknown slope function $\phi(t)$ in Eq. (55a), we follow the general procedure outlined in the Part I paper. For the sake of clarity and completeness, each step is presented below and particularized to the problem at hand (see Figs. 1 and 2).

7.1 Normalization. By the change of variables

$$
t=(d-c) s / 2+(c+d) / 2 \text { and } x=(d-c) r / 2+(c+d) / 2
$$

the crack surface $(c, d)$ can be converted into $(-1,1)$, and the main integral equation $(55 a)$ can be rewritten in normalized form

$$
\begin{aligned}
\frac{1}{\pi} f_{-1}^{1} & \left\{\frac{-2(\ell / a)^{2}}{(s-r)^{3}}-\frac{3(\ell / a)^{2}(a \beta)}{2(s-r)^{2}}\right. \\
& \left.+\frac{1-3(\ell / a)^{2}(a \beta)^{2} / 8-\left[\left(\ell^{\prime} / a\right) /(2 \ell / a)\right]^{2}}{s-r}+\mathcal{K}(r, s)\right\} \Phi(s) d s \\
& +\frac{\ell^{\prime} / a}{2} \Phi^{\prime}(r)+\frac{(a \beta)\left(\ell^{\prime} / a\right)}{2} \Phi(r)=\frac{P(r)}{G_{0}} e^{-\beta[a r+(c+d) / 2]}, \quad|r|<1
\end{aligned}
$$

where

$$
\begin{gathered}
a=(d-c) / 2=\text { half of the crack length } \\
\Phi(r)=\phi(a r+(c+d) / 2), \quad P(r)=p(a r+(c+d) / 2) \\
\mathcal{K}(r, s)=a k(a r+(c+d) / 2, a s+(c+d) / 2)
\end{gathered}
$$

and $k(x, t)$ is described by Eq. (56).

Note that in Eq. (58), $G(x)$ has been written as

$$
G(x)=G_{0} e^{\beta x}=G_{0} e^{\beta[((d-c) / 2) r+[(c+d) / 2]]}=G_{0} e^{(a \beta) r}\left(e^{a \beta}\right)^{((d+c) /(d-c))}
$$

where $a \beta=(d-c) \beta / 2$ and $(d+c) /(d-c)$ are two dimensionless quantities. Together with the terms $\ell / a$ and $\ell^{\prime} / a$ that appear in Eq. (58), the following dimensionless parameters are defined:

$$
\tilde{\ell}=\ell / a, \quad \tilde{\ell}^{\prime}=\ell^{\prime} / a \text { and } \tilde{\beta}=a \beta
$$

They will be used in the numerical implementation and results.

7.2 Representation of the Density Function. To proceed with the numerical approximation, a representation of $\Phi(s)$ is cho- 
sen so that one can evaluate the hypersingular and the Cauchy singular integrals by finite part and Cauchy principal value, respectively (see Refs. [11,12]). For the cubic hypersingular integral equation (58), the solution $\Phi(s)$ can be represented as

$$
\Phi(s)=g(s) \sqrt{1-s^{2}}
$$

where $g( \pm 1)$ is finite and $g( \pm 1) \neq 0[5]$. By finding numerical solution for $g(s)$, one can find the approximate solution for $\Phi(s)$. The representation (63) of $\Phi(s)$ is suggested by the following asymptotic behavior of the solution around the crack tips:

$$
\text { Displacements } \sim r^{3 / 2}, \quad \text { strains } \sim \sqrt{r}, \quad \text { stresses } \sim r^{-3 / 2}
$$

reported in Refs. [1,5].

7.3 Chebyshev Polynomial Expansion. In view of Eq. (63) and the fact that $\left\{U_{n}(s)\right\}$ are orthogonal on $(-1,1)$ with respect to the weight function $\sqrt{1-s^{2}}, g(s)$ can be most naturally expressed in terms of the Chebyshev polynomials of the second kind $U_{n}(s)$. However, the orthogonality is not required in the implementation of the numerical procedures, and either Chebyshev polynomials of the first kind $T_{n}(s)$ or of the second kind $U_{n}(s)$ may be employed in the approximation, i.e.,

$$
g(s)=\sum_{n=0}^{\infty} a_{n} T_{n}(s) \quad \text { or } g(s)=\sum_{n=0}^{\infty} A_{n} U_{n}(s)
$$

The coefficients $a_{n}$ 's or $A_{n}$ 's are numerically determined by the collocation method. As shown by Chan et al. [6], the two expansions should lead to consistent numerical results. In this paper, the expansion in terms of $U_{n}(s)$ is adopted, i.e.,

$$
\Phi(s)=\sqrt{1-s^{2}} \sum_{n=0}^{\infty} A_{n} U_{n}(s)
$$

where $U_{n}(s)$ is defined, as usual, by

$$
U_{n}(s)=\frac{\sin \left[(n+1) \cos ^{-1}(s)\right]}{\sin \left[\cos ^{-1}(s)\right]}, \quad n=0,1,2, \ldots
$$

Note that the single-valuedness condition (41) or, equivalently, $\int_{-1}^{1} \Phi(s) d s=0$ implies

$$
A_{0}=0
$$

Thus, the running index $n$ in Eq. (66) can start from 1 instead of 0 .

7.4 Evaluation of the Derivative of the Density Function. The term $\Phi^{\prime}(r)$ in Eq. (58) is evaluated using the expansion (66) and the fact that

$$
\frac{d}{d r}\left[U_{n}(r) \sqrt{1-r^{2}}\right]=-\frac{n+1}{\sqrt{1-r^{2}}} T_{n+1}(r), \quad n \geqslant 0
$$

Thus

$$
\Phi^{\prime}(r)=\frac{d}{d r}\left[\sqrt{1-r^{2}} \sum_{n=0}^{\infty} A_{n} U_{n}(r)\right]=\frac{-1}{\sqrt{1-r^{2}}} \sum_{n=0}^{\infty}(n+1) A_{n} T_{n}(r)
$$

7.5 Formation of the Linear System of Equations. The $A_{n}$ coefficients are determined by transforming the integral equation $(55 a)$ into a system of linear algebraic equations in terms of the $A_{n}$ 's. By replacing $\Phi(s)$ in Eq. (58) by the representation (66), and using Eq. (70), one obtains the governing integral equation in discretized form

$$
\begin{aligned}
-2 \widetilde{\ell}^{2} \sum_{n=1}^{\infty} \frac{A_{n}}{\pi} \int_{-1}^{1} \frac{U_{n}(s) \sqrt{1-s^{2}}}{(s-r)^{3}} d s-\frac{3 \widetilde{\ell}^{2} \widetilde{\beta}}{2} \sum_{n=1}^{\infty} \frac{A_{n}}{\pi} \int_{-1}^{1} \frac{U_{n}(s) \sqrt{1-s^{2}}}{(s-r)^{2}} d s \\
+\left[1-\frac{3 \widetilde{\ell}^{2} \widetilde{\beta}^{2}}{8}-\left(\frac{\tilde{\ell}^{\prime}}{2 \widetilde{\ell}}\right)^{2}\right] \sum_{n=1}^{\infty} \frac{A_{n}}{\pi} \int_{-1}^{1} \frac{U_{n}(s) \sqrt{1-s^{2}}}{s-r} d s \\
\quad+\sum_{n=1}^{\infty} \frac{A_{n}}{\pi} \int_{-1}^{1} \sqrt{1-s^{2}} U_{n}(s) \mathcal{K}(r, s) d s \\
\quad-\frac{\tilde{\ell}^{\prime}}{2 \sqrt{1-r^{2}}} \sum_{n=1}^{\infty}(n+1) A_{n} T_{n+1}(r)+\frac{\widetilde{\ell^{\prime}} \tilde{\beta}}{2} \sqrt{1-r^{2}} \sum_{n=1}^{\infty} A_{n} U_{n}(r) \\
=\frac{\mathcal{P}(r)}{G(r)}, \quad|r|<1
\end{aligned}
$$

We have used the running index $n$ that starts from 1 (see Eq. (68)).

7.6 Evaluation of Singular and Hypersingular Integrals. Formulas for evaluating singular integral terms in Eq. (71) are listed below:

$$
\begin{aligned}
& \frac{1}{\pi} \int_{-1}^{1} \frac{U_{n}(s) \sqrt{1-s^{2}}}{s-r} d s=-T_{n+1}(r), \quad|r|<1, \quad n \in \mathrm{Z}^{+} \\
& \frac{1}{\pi} \int_{-1}^{1} \frac{U_{n}(s) \sqrt{1-s^{2}}}{(s-r)^{2}} d s=-(n+1) U_{n}(r), \quad|r|<1, \quad n \in \mathrm{Z}^{+}
\end{aligned}
$$

$$
\begin{aligned}
& \frac{1}{\pi} \int_{-1}^{1} \frac{U_{n}(s) \sqrt{1-s^{2}}}{(s-r)^{3}} d s \\
& \quad=\left\{\begin{array}{cll}
-1, & n=0 \\
\frac{\left(n^{2}+n\right) U_{n+1}(r)-\left(n^{2}+3 n+2\right) U_{n-1}(r)}{4\left(1-r^{2}\right)}, & n \geqslant 1 & |r|<1
\end{array}\right.
\end{aligned}
$$

The details of the calculation can be found in Ref. [13].

7.7 Evaluation of Nonsingular Integral. By combining all the results obtained so far in the numerical approximation, one may rewrite Eq. (71) in the following form:

$$
\begin{aligned}
& \frac{-\tilde{\ell}^{2}}{2\left(1-r^{2}\right)} \sum_{n=1}^{\infty} A_{n}\left[\left(n^{2}+n\right) U_{n+1}(r)-\left(n^{2}+3 n+2\right) U_{n-1}(r)\right] \\
& \quad+\frac{3 \tilde{\beta} \widetilde{\ell}^{2}}{2} \sum_{n=1}^{\infty}(n+1) A_{n} U_{n}(r)-\left[1-\frac{3 \widetilde{\ell}^{2} \widetilde{\beta}^{2}}{8}-\left(\frac{\tilde{\ell}^{\prime}}{2 \tilde{\ell}}\right)^{2}\right] \\
& \quad \times \sum_{n=1}^{\infty} A_{n} T_{n+1}(r)+\sum_{n=1}^{\infty} \frac{A_{n}}{\pi} \int_{-1}^{1} \sqrt{1-s^{2}} U_{n}(s) \mathcal{K}(r, s) d s \\
& \quad-\frac{\tilde{\ell}^{\prime}}{2 \sqrt{1-r^{2}}} \sum_{n=1}^{\infty}(n+1) A_{n} T_{n+1}(r)+\frac{\tilde{\ell}^{\prime} \tilde{\beta}}{2} \sqrt{1-r^{2}} \sum_{n=1}^{\infty} A_{n} U_{n}(r) \\
& =\frac{\mathcal{P}(r)}{G(r)}, \quad|r|<1
\end{aligned}
$$

The regular kernel in Eq. (75) is actually a double integral, i.e., 


$$
\begin{aligned}
\int_{-1}^{1} \sqrt{1-s^{2}} U_{n}(s) \mathcal{K}(r, s) d s & =\int_{-1}^{1} \sqrt{1-s^{2}} U_{n}(s) a k(a r, a s) d s \\
& =\int_{-1}^{1} \sqrt{1-s^{2}} U_{n}(s) \\
& \times \int_{0}^{\infty} a N(\xi, 0) \sin [a \xi(s-r)] d \xi d s
\end{aligned}
$$

The Fourier sine transform in Eq. (76) can be efficiently evaluated by applying fast Fourier transform (FFT) [14]. The integral along $[-1,1]$ can be readily obtained by the Gaussian quadrature method [15].

\section{Stress Intensity Factors}

In classical LEFM, the SIFs are defined by

$$
K_{\mathrm{III}}^{C}(d)=\lim _{x \rightarrow d^{+}} \sqrt{2 \pi(x-d)} \sigma_{y z}(x, 0) \quad(x>d)
$$

and

$$
K_{\mathrm{III}}^{C}(c)=\lim _{x \rightarrow c^{-}} \sqrt{2 \pi(c-x)} \sigma_{y z}(x, 0) \quad(x<c)
$$

After normalization, the crack surfaces are located in the interval $(-1,1)$. The density function $\Phi(t)$ is expanded in terms of Chebyshev polynomials of the second kind $U_{n}$, which, when substituted into Eq. (55b), give rise to the following formulas for $|r|>1$ (see Ref. [13]):

$$
\begin{aligned}
& \frac{1}{\pi} \int_{-1}^{1} \frac{U_{n}(s) \sqrt{1-s^{2}}}{s-r} d s=-\left(r-\frac{|r|}{r} \sqrt{r^{2}-1}\right)^{n+1}, \quad n \geqslant 0 \\
& \frac{1}{\pi} \int_{-1}^{1} \frac{U_{n}(s) \sqrt{1-s^{2}}}{(s-r)^{2}} d s=-(n+1)\left(1-\frac{|r|}{\sqrt{r^{2}-1}}\right) \\
& \times\left(r-\frac{|r|}{r} \sqrt{r^{2}-1}\right)^{n}, \quad n \geqslant 0 \\
& \frac{1}{\pi} \int_{-1}^{1} \frac{U_{n}(s) \sqrt{1-s^{2}}}{(s-r)^{3}} d s \\
& =\frac{-1}{2}(n+1)\left(r-\frac{|r|}{r} \sqrt{r^{2}-1}\right)^{n-1} \\
& \times\left[n\left(1-\frac{|r|}{\sqrt{r^{2}-1}}\right)^{2}+\frac{r-\frac{|r|}{r} \sqrt{r^{2}-1}}{\left(\sqrt{r^{2}-1}\right)^{3}}\right], \quad n \geqslant 0
\end{aligned}
$$

The highest singularity in Eqs. (79)-(81) appears in the last term in Eq. (81) and it behaves like $\left(r^{2}-1\right)^{-3 / 2}$ as $r \rightarrow 1^{+}$or $r \rightarrow$ $-1^{-}$. Motivated by such asymptotic behavior, we define the SIFs for strain-gradient elasticity as

$$
\begin{aligned}
& \ell K_{\mathrm{III}}(d)=\lim _{x \rightarrow d^{+}} 2 \sqrt{2 \pi(x-d)}(x-d) \sigma_{y z}(x, 0) \quad(x>d) \\
& \ell K_{\mathrm{III}}(c)=\lim _{x \rightarrow c^{-}} 2 \sqrt{2 \pi(c-x)}(c-x) \sigma_{y z}(x, 0) \quad(x<c)
\end{aligned}
$$

Thus,

$$
\begin{aligned}
\ell K_{\mathrm{III}}(d)= & \lim _{x \rightarrow d^{+}} 2 \sqrt{2 \pi(x-d)}(x-d) \sigma_{y z}(x, 0) \quad(x>d) \\
= & \lim _{r \rightarrow 1^{+}} 2 \sqrt{2 \pi\left[\left(\frac{d-c}{2}\right) r+\frac{c+d}{2}-d\right]}(a r-a) \\
& \times \sigma_{y z}\left(\frac{d-c}{2} r+\frac{c+d}{2}, 0\right) \quad(r>1) \\
= & 2 a \sqrt{2 \pi a} \lim _{r \rightarrow 1^{+}} \sqrt{(r-1)}(r-1) \sigma_{y z}((d-c) r / 2 \\
& +(c+d) / 2,0) \quad(r>1) \\
= & 2 a \sqrt{2 \pi a} \lim _{r \rightarrow 1^{+}} \sqrt{(r-1)}(r-1) G_{0} e^{a \beta r} e^{\beta(d+c) / 2} \\
& \times\left(\frac{-2 \ell^{2}}{\pi a^{2}}\right) \int_{-1}^{1} \frac{\Phi(s)}{(s-r)^{3}} d s \quad(r>1)
\end{aligned}
$$

By using Eq. (66) in conjunction with Eq. (81), we obtain from Eq. (84)

$$
\begin{aligned}
K_{\mathrm{III}}(d)= & 2 \sqrt{2 \pi a}\left(\frac{-2 \ell}{a}\right) G_{0} e^{\beta d} \lim _{r \rightarrow 1^{+}}(r-1)^{3 / 2} \\
& \times \sum_{n=0}^{N} \frac{-(n+1)}{2}\left(r-\frac{|r|}{r} \sqrt{r^{2}-1}\right)^{n-1} \\
& \times\left[n\left(1-\frac{|r|}{\sqrt{r^{2}-1}}\right)^{2}+\frac{r-\frac{|r|}{r} \sqrt{r^{2}-1}}{\sqrt{r^{2}-1^{3}}}\right] A_{n} \\
= & \sqrt{\pi a} G_{0} e^{\beta d}(\ell / a) \sum_{n=0}^{\infty}(n+1) A_{n}
\end{aligned}
$$

Similarly,

$$
K_{\mathrm{III}}(c)=\sqrt{\pi a} G_{0} e^{\beta c}(\ell / a) \sum_{n=0}^{\infty}(-1)^{n}(n+1) A_{n}
$$

\section{Results and Discussion}

The numerical results include crack surface displacements, strains, stresses, and SIFs.

9.1 Crack Surface Displacements. The (normalized) crack surface displacements shown in Figs. 4-8 are obtained by integrating the slope function (see Eq. (87) below). Figure 4 shows a full normalized crack sliding displacement profile for a homogeneous medium $(\widetilde{\beta}=0)$ with the strain-gradient effect. The crack profile in Fig. 4 is symmetric because the material is homogeneous. Figures 5 and 6 are for classical LEFM. Figures 7 and 8 are for the strain-gradient theory. As $\beta<0$, the material has larger shear modulus at the left side of the crack than at the right side, and thus the material is stiffer on the left and more compliant on the right, as shown in Figs. 5 and 7. Similarly, Figs. 6 and 8 illustrate the case of $\beta>0$ and confirm that the material is stiffer on the right and more compliant on the left. The variation of the shear modulus destroys the symmetry of the displacement profiles. The most prominent feature is the cusping phenomena around the crack tips, as shown in Figs. 4, 7, and 8. The difference between Figs. 5 and 6 and Figs. 7 and 8 is the cusp at the crack tips. In Figs. 5 and 6, one may observe that the profiles have a tangent line with infinite slope at the crack tips, which is a common crack behavior exhibited in the classical LEFM. However, such is not the case in gradient theory as evidenced by the numerical results shown. 


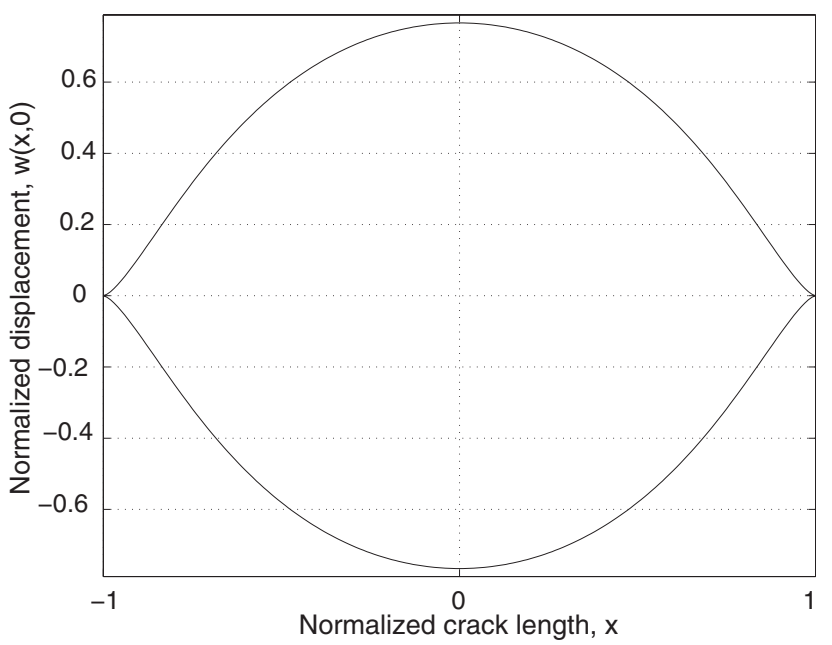

Fig. 4 Full crack displacement profile for homogeneous material $(\tilde{\beta}=0)$ under uniform crack surface shear loading $\sigma_{y z}(x, 0)$ $=-p_{0}$ with choice of (normalized) $\tilde{\ell}=0.2$ and $\tilde{\ell}^{\prime}=0$

9.2 Strains. We have used the strain-like field, $\phi(x)$ (the slope function), as the unknown density function in our integral equation formulation. The normalized version, $\Phi(x)$, with various $\tilde{\ell}$ is plotted in Fig. 9. Note that $\Phi( \pm 1)=0$ while in classical LEFM, $\Phi( \pm 1)= \pm \infty$. The vanishing slope is equivalent to the cusping at the crack tips. The (normalized) crack displacement profile $w(r, 0)$ can be obtained by

$$
w(r, 0)=\int_{-1}^{r} \Phi(s) d s=\int_{-1}^{r} \sqrt{1-s^{2}} \sum_{n=0}^{N} A_{n} U_{n}(s) d s
$$

As $\ell$ decreases, $\Phi(x)$ seems to converge to the slope function of the classical LEFM case in the region away from the crack tips, where $\Phi(x)$ is very different from its classical counterpart near the crack tips.

9.3 Stresses. Similar to classical LEFM, the stress $\sigma_{y z}(x, 0)$ diverges as $x$ approaches the crack tips along the ligament (Fig.

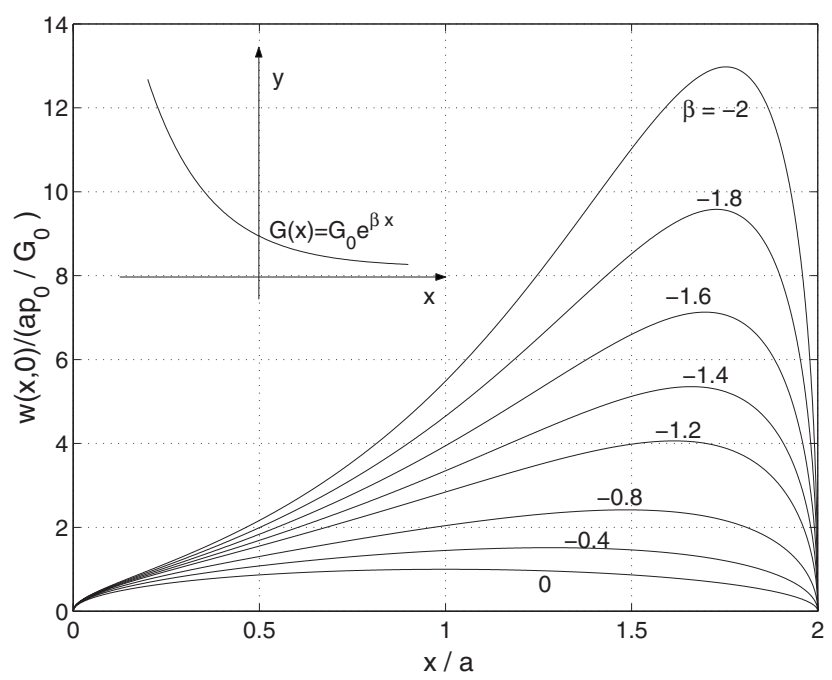

Fig. 5 Classical LEFM, i.e., $\tilde{\ell}=\tilde{\ell}^{\prime} \rightarrow 0$. Crack surface displacement in an infinite nonhomogeneous plane under uniform crack surface shear loading $\sigma_{y z}(x, 0)=-p_{0}$ and shear modulus $G(x)=G_{0} e^{\beta x}$. Here, $a=(d-c) / 2$ denotes the half crack length.

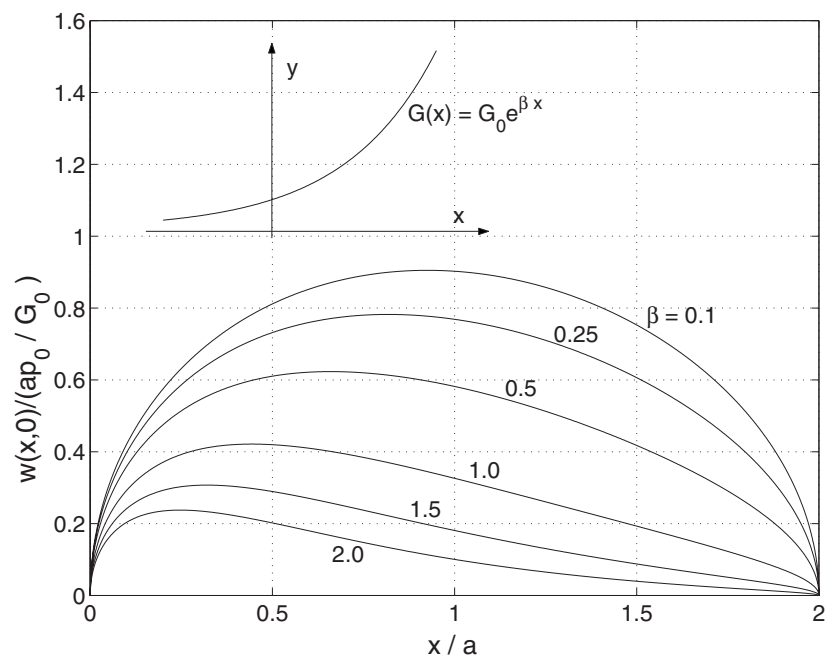

Fig. 6 Classical LEFM, i.e., $\tilde{\ell}=\tilde{\ell}^{\prime} \rightarrow 0$. Crack surface displacement in an infinite nonhomogeneous plane under uniform crack surface shear loading $\sigma_{y z}(x, 0)=-p_{0}$ and shear modulus $G(x)=G_{0} e^{\beta x}$. Here, $a=(d-c) / 2$ denotes the half crack length.

10). Moreover, the sign of the stress changes, and as $\ell$ decreases, the interior part (i.e., the region apart from the two crack tips) of $\sigma_{y z}(x, 0)$ seems to converge to the solution of classical LEFM. The finding of the negative near-tip stress is consistent with the results by Zhang et al. [8] who also investigated a Mode-III crack in elastic materials with strain-gradient effects; this negative stress may be considered as a necessity for the crack surface to reattach near the tips. The point worth noting here is that not all straingradient theories possess the negative-stress feature near the crack tips. For instance, the strain-gradient elasticity theory for cellular materials [16] and elastic-plastic materials with strain-gradient effects [17], which fall within the classical couple-stress theory framework, shows a positive-stress singularity near the crack tip. On the other hand, the strain-gradient theory proposed by Fleck and Hutchinson [18], which does not fall into the above framework, predicts a compressive stress near the tip of a tensile Mode-I crack [19,20].

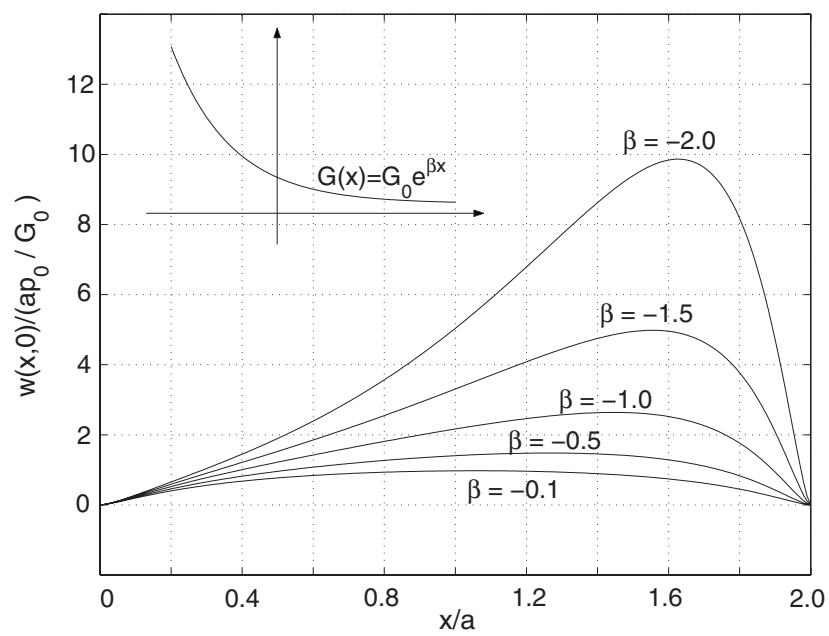

Fig. 7 Crack surface displacement in an infinite nonhomogeneous plane under uniform crack surface shear loading $\sigma_{y z}(x, 0)=-p_{0}$ and shear modulus $G(x)=G_{0} e^{\beta x}$ with choice of (normalized) $\tilde{\ell}=0.10$ and $\tilde{\ell}^{\prime}=0.01$. Here, $a=(d-c) / 2$ denotes the half crack length. 


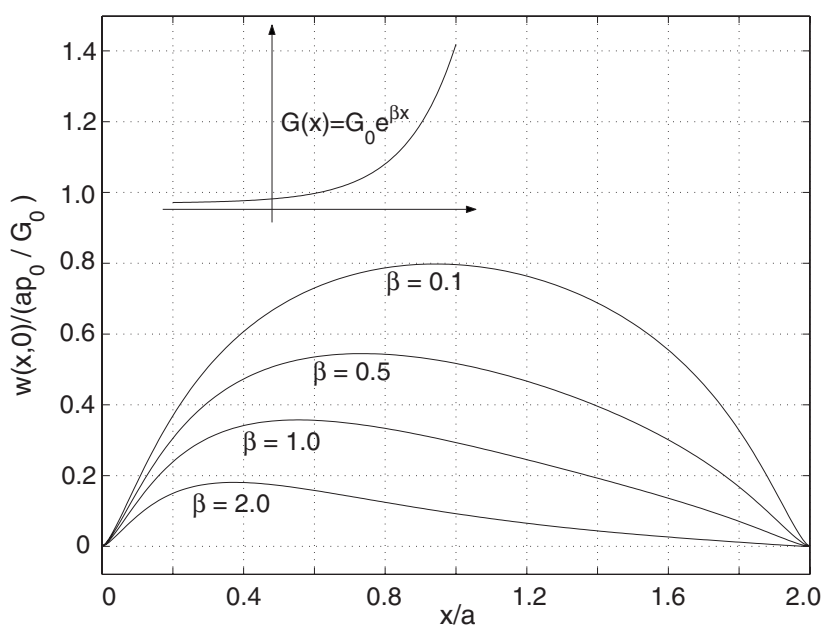

Fig. 8 Crack surface displacement in an infinite nonhomogeneous plane under uniform crack surface shear loading $\sigma_{y z}(x, 0)=-p_{0}$ and shear modulus $G(x)=G_{0} e^{\beta x}$ with choice of (normalized) $\tilde{\ell}=0.10$ and $\tilde{\ell}^{\prime}=0.01$. Here, $a=(d-c) / 2$ denotes the half crack length.

9.4 Stress Intensity Factors. Besides using $\phi(x)$ as the unknown density function, one may also use displacement $w(x)$ to be the unknown in the formulation of the integral equation (see Appendix). By rewriting $K_{\mathrm{III}}^{C}$ in terms of the coefficients in the expansion for $w$, one obtains (see Ref. [6]) the following.

- With $T_{n}$ expansion,

$$
\begin{gathered}
\frac{K_{\mathrm{III}}^{C}(c)}{G_{0} \sqrt{\pi(d-c) / 2}}=e^{\beta c} \sum_{0}^{N}(-1)^{n} a_{n} \\
\frac{K_{\mathrm{III}}^{C}(d)}{G_{0} \sqrt{\pi(d-c) / 2}}=e^{\beta d} \sum_{0}^{N} a_{n}
\end{gathered}
$$

- With $U_{n}$ expansion,

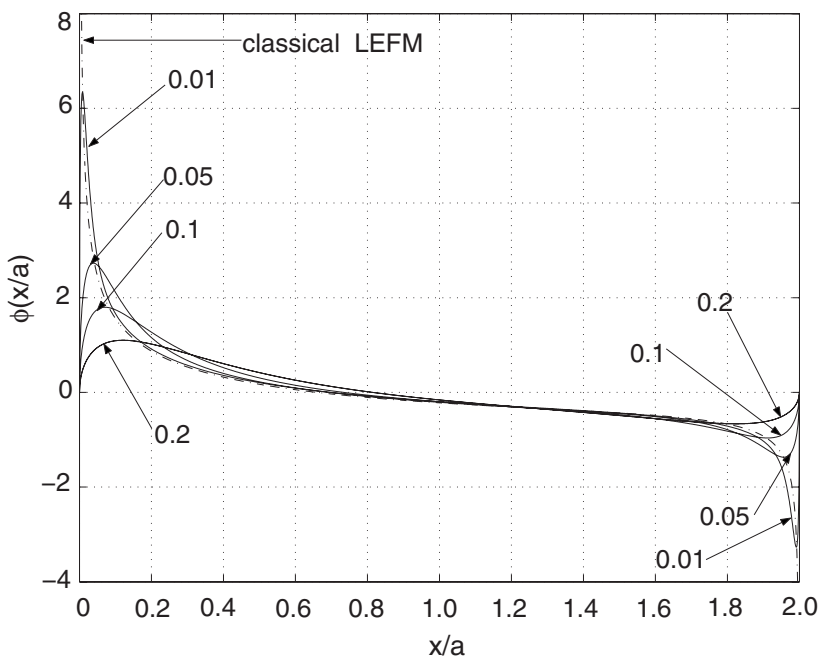

Fig. 9 Strain $\phi(x / a)$ along the crack surface $(c, d)=(0,2)$ for $\tilde{\beta}=0.5, \tilde{\ell}^{\prime}=0$, and various $\tilde{\ell}$ in an infinite nonhomogeneous plane under uniform crack surface shear loading $\sigma_{y z}(x, 0)=$ $-p_{0}$ and shear modulus $G(x)=G_{0} e^{\beta x}$. Here, $a=(d-c) / 2$ denotes the half crack length.

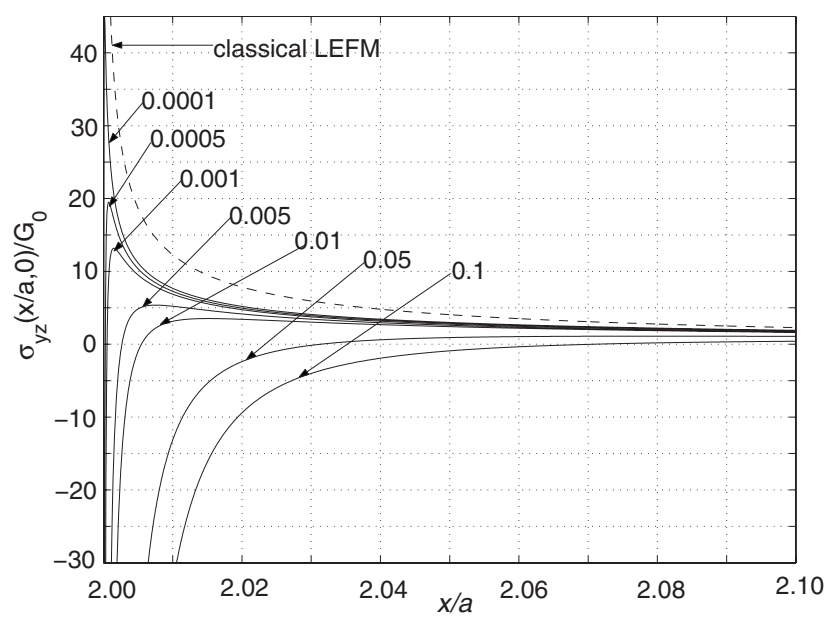

Fig. 10 Stress $\sigma_{y z}(x / a, 0) / G_{0}$ along the ligament for $\tilde{\beta}=0.5, \tilde{\ell}^{\prime}$ $=0$, and various $\tilde{\ell}$. Crack surface $(c, d)=(0,2)$ located in an infinite nonhomogeneous plane is assumed to be under uniform crack surface shear loading $\sigma_{y z}(x, 0)=-p_{0}$ and shear modulus $G(x)=G_{0} e^{\beta x}$. Here, $a=(d-c) / 2$ denotes the half crack length.

$$
\begin{gathered}
\frac{K_{\mathrm{III}}(c)}{G_{0} \sqrt{\pi(d-c) / 2}}=e^{\beta c} \sum_{0}^{N}(-1)^{n}(n+1) A_{n} \\
\frac{K_{\mathrm{III}}(d)}{G_{0} \sqrt{\pi(d-c) / 2}}=e^{\beta d} \sum_{0}^{N}(n+1) A_{n}
\end{gathered}
$$

Table 3 contains the (normalized) SIFs for the case of classical LEFM by using both $T_{n}$ and $U_{n}$ expansions [see Eqs. (63) and (65)]. The SIFs in Table 3 have been obtained by using Eqs. (88) and (89), and they are close to the results reported by Erdogan [7].

Table 4 contains the SIFs for strain-gradient elasticity at $\tilde{\ell}$ $=0.1$ and $\tilde{\ell}^{\prime}=0.01$. One observes that the dependence of $K_{\mathrm{III}}(c)$ and $K_{\mathrm{III}}(d)$ is similar to the classical case reported in Table 3.

\section{Concluding Remarks}

This paper has shown that the integral equation method is an effective means of formulating crack problems for a FGM considering strain-gradient effects. The theoretical framework and numerical analysis has been utilized to solve antiplane shear crack problems in FGMs by using Casal's continuum. The behavior of

Table 3 Normalized SIFs for Mode-III crack problem in a FGM $\left(\ell=\ell^{\prime} \rightarrow 0\right)$

\begin{tabular}{rccccc}
\hline \hline & \multicolumn{2}{c}{$U_{n}$ representation } & & \multicolumn{2}{c}{$T_{n}$ representation } \\
\cline { 2 - 3 } \cline { 5 - 6 }$\beta\left(\frac{d-c}{2}\right)$ & $\frac{K_{\mathrm{III}}(c)}{p_{0} \sqrt{\pi(d-c) / 2}}$ & $\frac{K_{\mathrm{III}}(d)}{p_{0} \sqrt{\pi(d-c) / 2}}$ & & $\frac{K_{\mathrm{III}}(c)}{p_{0} \sqrt{\pi(d-c) / 2}}$ & $\frac{K_{\mathrm{III}}(d)}{p_{0} \sqrt{\pi(d-c) / 2}}$ \\
\hline-2.00 & 1.21779 & 0.55672 & & 1.21779 & 0.55672 \\
-1.50 & 1.17801 & 0.63007 & & 1.17801 & \\
-1.00 & 1.14307 & 0.72845 & & 1.14307 & 0.728007 \\
-0.50 & 1.09036 & 0.85676 & & 1.09036 & 0.85676 \\
-0.10 & 1.02289 & 0.97312 & & 1.02289 & 0.97312 \\
0.00 & 1.00000 & 1.00000 & & 1.00000 & 1.00000 \\
0.10 & 0.97312 & 1.02289 & & 0.97312 & 1.02289 \\
0.50 & 0.85676 & 1.09036 & & 0.85676 & 1.09036 \\
1.00 & 0.72845 & 1.14307 & & 0.72845 & 1.14307 \\
1.50 & 0.63007 & 1.17801 & & 0.63007 & 1.17801 \\
2.00 & 0.55672 & 1.21779 & & 0.55672 & 1.21779 \\
\hline \hline
\end{tabular}


Table 4 Normalized generalized SIFs for a Mode-III crack at $\tilde{\ell}$ $=0.1, \tilde{\ell}=0.01$, and various values of $\tilde{\boldsymbol{\beta}}$

\begin{tabular}{ccc}
\hline \hline$\tilde{\beta}$ & $\frac{K_{\mathrm{III}}(c)}{p_{0} \sqrt{\pi(d-c) / 2}}$ & $\frac{K_{\mathrm{III}}(d)}{p_{0} \sqrt{\pi(d-c) / 2}}$ \\
\hline-2.00 & 1.23969 & \\
-1.00 & 1.12585 & 0.49938 \\
-0.50 & 1.04849 & 0.67600 \\
-0.10 & 0.96814 & 0.80248 \\
0.00 & 0.94385 & 0.91658 \\
0.10 & 0.91677 & 0.94385 \\
0.50 & 0.80277 & 0.96828 \\
1.00 & 0.67637 & 1.04854 \\
2.00 & 0.49938 & 1.12584 \\
\hline \hline
\end{tabular}

the solution around the crack tips is affected by the strain-gradient theory, and not by the gradation of the materials. Also, the integral equation formulation has been found to be an adequate tool for implementing the numerical procedures and to assess physical quantities such as crack surface displacements, strains, stresses, and SIFs. Further experiments are needed for justifying the physical aspects of the method. Future work includes extension of the theory to Mode-I crack problems.

\section{Acknowledgment}

G.H.P. would like to thank the support from the National Science Foundation under Grant No. CMS 0115954 (Mechanics and Materials Program) and from the NASA-Ames "Engineering for Complex Systems Program," and the NASA-Ames Chief Engineer (Dr. Tina Panontin) through Grant No. NAG 2-1424.

A.C.F. acknowledges the support from the USA National Science Foundation (NSF) through Grant No. DMS-9971322 and UC Davis Chancellor's Fellowship.

Y.-S.C. thanks the support from the Applied Mathematical Sciences Research Program of the Office of Mathematical, Information, and Computational Sciences, U.S. Department of Energy, under Contract No. DE-AC05-00OR22725 with UT-Battelle, LLC; he also thanks the grant (No. W911NF-05-1-0029) from the U.S. Department of Defense, Army Research Office. Organized Research Award (2007) from University of Houston-Downtown is also acknowledged by Y.-S.C.

\section{Appendix: Hierarchy of Governing Integral Equations}

In this appendix, we list the type of the physical problem under antiplane shear loading, its governing PDE, and integral equation associated with the choice of the density function. The corresponding references in the literature are also provided.

1. Classical LEFM, Homogeneous Materials $\left(G \equiv G_{0}\right)$

- PDE: Laplace equation $\nabla^{2} w(x, y)=0$.

- Integral equation with the density function $\phi(x)$ $=\partial w(x, 0) / \partial x$ :

$$
\frac{G_{0}}{\pi} \int_{c}^{d} \frac{\phi(t)}{t-x} d t=p(x), \quad c<x<d
$$

- Integral equation with the density function $\phi(x)$ $=w(x, 0)$ :

$$
\frac{G_{0}}{\pi} \int_{c}^{d} \frac{\phi(t)}{(t-x)^{2}} d t=p(x), \quad c<x<d
$$

Many standard textbooks have covered the Laplace equation (see, for example, Ref. [21]).
2. Classical LEFM, Nonhomogeneous Materials $(G \equiv G(y)$ $\left.=G_{0} e^{\gamma y}\right)$

- PDE: Perturbed Laplace equation $\left(\nabla^{2}+\gamma(\partial / \partial y)\right) w(x, y)$ $=0$.

- Integral equation with the density function $\phi(x)$ $=\partial w(x, 0) / \partial x$ :

$$
\frac{G_{0}}{\pi} \int_{-a}^{a}\left[\frac{1}{t-x}+\widetilde{K}_{\gamma}(x, t)\right] \phi(t) d t=p(x), \quad-a<x<a
$$

Erdogan and Ozturk [22] have investigated this problem as bonded nonhomogeneous materials with an interface cut.

3. Classical LEFM, Nonhomogeneous Materials $(G \equiv G(x)$ $\left.=G_{0} e^{\beta x}\right)$

- PDE: Perturbed Laplace equation $\left(\nabla^{2}+\beta(\partial / \partial x)\right) w(x, y)$ $=0$.

- Integral equation with the density function $\phi(x)$ $=\partial w(x, 0) / \partial x$ :

$$
\begin{aligned}
& \frac{G(x)}{\pi} \int_{c}^{d}\left[\frac{1}{t-x}+\frac{\beta}{2} \log |t-x|+\tilde{N}(x, t)\right] \phi(t) d t \\
& \quad=p(x), \quad c<x<d
\end{aligned}
$$

- Integral equation with the density function $\phi(x)$ $=w(x, 0)$ :

$$
\begin{aligned}
& \frac{G(x)}{\pi} f_{c}^{d}\left[\frac{1}{(t-x)^{2}}+\frac{\beta}{2(t-x)}+N(x, t)\right] \phi(t) d t \\
& \quad=p(x), \quad c<x<d
\end{aligned}
$$

The regular kernels $\tilde{N}(x, t)$ in Eq. (A4) and $N(x, t)$ in Eq. (A5) can be found in Ref. [6]. Erdogan [7] has studied this problem for bonded nonhomogeneous materials.

4. Gradient Elasticity, Homogeneous Materials $\left(G \equiv G_{0}\right)$

- PDE: Helmholtz-Laplace equation $\left(1-\ell^{2} \nabla^{2}\right) \nabla^{2} w(x, y)$ $=0$.

- Integral equation with the density function $\phi(x)$ $=\partial w(x, 0) / \partial x$ :

$$
\begin{aligned}
\frac{1}{\pi} \int_{c}^{d} & \left\{\frac{-2 \ell^{2}}{(t-x)^{3}}+\frac{1-\left(\ell^{\prime} / \ell\right)^{2} / 4}{t-x}+K_{0}(t-x)\right\} \phi(t) d t \\
& +\frac{\ell^{\prime}}{2} \phi^{\prime}(x)=\frac{p(x)}{G_{0}}
\end{aligned}
$$

Fannjiang et al. [5] have studied Eq. (A6) in detail.

5. Gradient Elasticity, Nonhomogeneous Materials $(G \equiv G(y)$ $\left.=G_{0} e^{\gamma y}\right)$

- PDE: $\left(1-\gamma \ell^{2}(\partial / \partial y)-\ell^{2} \nabla^{2}\right)\left(\nabla^{2}+\gamma(\partial / \partial y)\right) w(x, y)=0$.

- Integral equation with the density function $\phi(x)$ $=\partial w(x, 0) / \partial x$ : 


$$
\begin{aligned}
\frac{G_{0}}{\pi} \int_{-a}^{a} & \left\{\frac{-2 \ell^{2}}{(t-x)^{3}}+\frac{5 \ell^{2} \gamma^{2} / 8+\ell^{\prime} \gamma / 4+1-\left(\ell^{\prime} / \ell\right)^{2} / 4}{t-x}\right. \\
& \left.+k_{\gamma}(x, t)\right\} \phi(t) d t+G\left(\ell^{\prime} / 2+\ell^{2} \gamma\right) \phi^{\prime}(x) \\
& =p(x), \quad|x|<a
\end{aligned}
$$

This is the Part I paper by Paulino et al. [1].

6. Gradient Elasticity, Nonhomogeneous Materials $(G \equiv G(x)$ $\left.=G_{0} e^{\beta x}\right)$

- PDE: $\left(1-\beta \ell^{2}(\partial / \partial x)-\ell^{2} \nabla^{2}\right)\left(\nabla^{2}+\beta(\partial / \partial x)\right) w(x, y)=0$.

- Integral equation with the density function $\phi(x)$ $=\partial w(x, 0) / \partial x$ :

$$
\begin{aligned}
\frac{1}{\pi} \int_{c}{ }_{c}^{d} & \left\{\frac{-2 \ell^{2}}{(t-x)^{3}}-\frac{3 \beta \ell^{2}}{2(t-x)^{2}}+\frac{1-3 \ell^{2} \beta^{2} / 8-\left[\ell^{\prime} /(2 \ell)\right]^{2}}{t-x}\right. \\
& +k(x, t)\} \phi(t) d t+\frac{\ell^{\prime}}{2} \phi^{\prime}(x)+\frac{\beta \ell^{\prime}}{2} \phi(x) \\
= & p(x) / G, \quad c<x<d
\end{aligned}
$$

This is the main governing integral equation (55a).

- Integral equation with the density function $\phi(x)$ $=w(x, 0)$ :

$$
\begin{aligned}
\frac{1}{\pi} f_{c}^{d} & \left\{\frac{-6 \ell^{2}}{(t-x)^{4}}-\frac{3 \ell^{2} \beta}{(t-x)^{3}}+\frac{1-\left(\frac{\ell^{\prime}}{2 \ell}\right)^{2}-\frac{3 \ell^{2} \beta^{2}}{8}}{(t-x)^{2}}\right. \\
& \left.+\frac{\frac{\beta}{2}\left[1-\left(\frac{\ell^{\prime}}{2 \ell}\right)^{2}\right]+\frac{\ell^{2} \beta^{3}}{16}}{t-x}+\tilde{k}(x, t)\right\} \phi(t) d t \\
& +\frac{\ell^{\prime}}{2} \phi^{\prime \prime}(x)-\frac{\ell^{\prime} \beta}{2} \phi^{\prime}(x)-\left[\frac{1}{\ell}\left(\frac{\ell^{\prime}}{2 \ell}\right)^{3}+\frac{\ell^{\prime}}{8 \ell^{2}}\right] \phi(x) \\
= & \frac{p(x)}{G(x)}, \quad c<x<d
\end{aligned}
$$

\section{References}

[1] Paulino, G. H., Chan, Y.-S., and Fannjiang, A. C., 2003, "Gradient Elasticity Theory for Mode III Fracture in Functionally Graded Materials-Part I: Crack
Perpendicular to the Material Gradation,” ASME J. Appl. Mech., 70(4), pp. 531-542.

[2] Chan, Y.-S., Paulino, G. H., and Fannjiang, A. C., 2006, "Change of Constitutive Relations Due to Interaction Between Strain-Gradient Effect and Material Gradation," ASME J. Appl. Mech., 73(5), pp. 871-875.

[3] Exadaktylos, G., Vardoulakis, I., and Aifantis, E., 1996, "Cracks in Gradient Elastic Bodies With Surface Energy,” Int. J. Fract., 79(2), pp. 107-119.

[4] Vardoulakis, I., Exadaktylos, G., and Aifantis, E., 1996, "Gradient Elasticity With Surface Energy: Mode-III Crack Problem,” Int. J. Solids Struct., 33(30), pp. 4531-4559.

[5] Fannjiang, A. C., Chan, Y.-S., and Paulino, G. H., 2002, "Strain-Gradient Elasticity for Mode III Cracks: A Hypersingular Integrodifferential Equation Approach,” SIAM J. Appl. Math., 62(3), pp. 1066-1091.

[6] Chan, Y.-S., Paulino, G. H., and Fannjiang, A. C., 2001, "The Crack Problem for Nonhomogeneous Materials Under Antiplane Shear Loading-A Displacement Based Formulation,” Int. J. Solids Struct., 38(17), pp. 2989-3005.

[7] Erdogan, F., 1985, "The Crack Problem for Bonded Nonhomogeneous Materials Under Antiplane Shear Loading," ASME J. Appl. Mech., 52(4), pp. 823828.

[8] Zhang, L., Huang, Y., Chen, J. Y., and Hwang, K. C., 1998, "The Mode III Full-Field Solution in Elastic Materials With Strain Gradient Effects," Int. J. Fract., 92(4), pp. 325-348.

[9] Georgiadis, H. G., 2003, "The Mode III Crack Problem in Microstructured Solids Governed by Dipolar Gradient Elasticity: Static and Dynamic Analysis," ASME J. Appl. Mech., 70(4), pp. 517-530.

[10] Titchmarsh, E. C., 1986, Introduction to the Theory of Fourier Integrals, Chelsea, New York.

[11] Kaya, A. C., and Erdogan, F., 1987, "On the Solution of Integral Equations With Strongly Singular Kernels," Q. Appl. Math., 45(1), pp. 105-122.

[12] Monegato, G., 1994, "Numerical Evaluation of Hypersingular Integrals," J. Comput. Appl. Math., 50, pp. 9-31.

[13] Chan, Y.-S., Fannjiang, A. C., and Paulino, G. H., 2003, "Integral Equations With Hypersingular Kernels-Theory and Application to Fracture Mechanics," Int. J. Eng. Sci., 41, pp. 683-720.

[14] Folland, G. B., 1992, Fourier Analysis and Its Applications, Wadsworth and Brooks/Cole Advanced Books and Software, Pacific Grove, CA.

[15] Stroud, A. H., and Secrest, D., 1966, Gaussian Quadrature Formulas, Prentice-Hall, New York

[16] Chen, J. Y., Huang, Y., Zhang, L., and Ortiz, M., 1998, "Fracture Analysis of Cellular Materials: A Strain Gradient Model,” J. Mech. Phys. Solids, 46(5), pp. 789-828.

[17] Huang, Y., Chen, J. Y., Guo, T. F., Zhang, L., and Hwang, K. C., 1999, “Analytic and Numerical Studies on Mode I and Mode II Fracture in Elastic-Plastic Materials With Strain Gradient Effects," Int. J. Fract., 100(1), pp. 1-27.

[18] Fleck, N. A., and Hutchinson, J. W., 1997, "Strain Gradient Plasticity," Advances in Applied Mechanics, Vol. 33, J. W. Hutchinson and T. Y. Wu, eds., Academic, New York, pp. 295-361.

[19] Chen, J. Y., Wei, Y., Huang, Y., Hutchinson, J. W., and Hwang, K. C., 1999, "The Crack Tip Fields in Strain Gradient Plasticity: The Asymptotic and Numerical Analyses," Eng. Fract. Mech., 64, pp. 625-648.

[20] Shi, M. X., Huang, Y., and Hwang, K. C., 2000, "Fracture in a Higher-Order Elastic Continuum," J. Mech. Phys. Solids, 48(12), pp. 2513-2538.

[21] Sneddon, I. N., 1972, The Use of Integral Transforms, McGraw-Hill, New York.

[22] Erdogan, F., and Ozturk, M., 1992, "Diffusion Problems in Bonded Nonhomogeneous Materials With an Interface Cut," Int. J. Eng. Sci., 30(10), pp. 15071523 